\title{
Live-Cell Imaging Evidence for the Ciliary Transport of Rod Photoreceptor Opsin by Heterotrimeric Kinesin-2
}

\author{
Deepti Trivedi, Emilie Colin, Carrie M. Louie, and David S. Williams \\ Departments of Ophthalmology and Neurobiology, Jules Stein Eye Institute, University of California, Los Angeles School of Medicine, Los Angeles, \\ California 90095
}

Primary cilia detect extracellular signals through membrane receptors and channels. The outer segment of a vertebrate photoreceptor cell represents the most elaborate of all primary cilia, containing extraordinarily large amounts of the visual receptor protein, opsin. Because of its high abundance, opsin represents a potential model system for the study of ciliary membrane receptors, including their transport. Here, we have analyzed the movement of ciliary opsin to test whether the highly conserved intraflagellar transport (IFT), as driven by heterotrimeric kinesin-2, is required. Results show that opsin can enter and move along the primary cilium of a nonphotoreceptor cell (an hTERT-RPE1 epithelial cell), suggesting that it can co-opt the basic anterograde motor system of cilia. Fluorescence recovery after photobleaching analysis of cilia of hTERTRPE1 cells showed that the movement of ciliary opsin was comparable to that of the IFT protein, IFT88. Moreover, the movement of opsin in these cilia, as well as in cilia of mouse rod photoreceptor cells, was reduced significantly when KIF3A, the obligate motor subunit of heterotrimeric kinesin-2, was deficient. These studies therefore provide evidence from live-cell analysis that the conserved heterotrimeric kinesin-2 is required for the normal transport of opsin along the ciliary plasma membrane.

\section{Introduction}

Primary cilia have important functions in many types of cells, such that cilium dysfunction causes a wide variety of diseases, known as ciliopathies, many of which are syndromic (Badano et al., 2005; Hildebrandt et al., 2011). The role of primary cilia is to detect extracellular signals through a receptor or channel that is integral to the ciliary plasma membrane. The movement of these transmembrane receptors or channels into the cilium and then along the cilium is therefore essential for ciliary function.

The most elaborate primary cilium is the outer segment of a photoreceptor cell. The receptor in this case is the light receptor, rhodopsin, which is packed at a very high density in a stack of disk membranes that form by amplification of the distal ciliary plasma membrane. The continuous renewal of the rhodopsincontaining disk membranes requires an extraordinary amount of trafficking to the cilium and along the ciliary axoneme to the site of disk membrane morphogenesis (Young, 1967, 1968; Papermaster et al., 1985; Deretic and Papermaster, 1991).

Received Jan. 2, 2012; revised May 22, 2012; accepted June 15, 2012.

Author contributions: D.T. and D.S.W. designed research; D.T., E.C., and C.M.L. performed research; D.T. and D.S.W. contributed unpublished reagents/analytic tools; D.T., E.C., C.M.L., and D.S.W. analyzed data; D.T. and D.S.W. wrote the paper.

The research was supported by National Institutes of Health (NIH) Grant EY13408 and NIH Core Grant EY00331. C.M.L. was supported by NIH Training Grant GM008243. D.S.W. is a Jules and Doris Stein RBP Professor. We thank those who kindly provided cells, gene constructs, and mouse lines. The hTERT-RPE1 cells were obtained from Dr. Erica Golemis. The Ift88-YFP and Arl13b-GFP constructs were provided by Drs. Jagesh Shah and Gregory Pazour, respectively. The RHO-EGFP knock-in and RPE65 ${ }^{-/-}$mice that were used for breeding in current study originated from Drs. Theodore Wensel and Michael Redmond, respectively; breeders were obtained from Drs. Wensel and Dean Bok, respectively.

Correspondence should be addressed to David S. Williams, Jules Stein Eye Institute, UCLA School of Medicine, 100 Stein Plaza, Los Angeles, CA 90095. E-mail: dswilliams@ucla.edu.

DOI:10.1523/JNEUROSCI.0015-12.2012

Copyright $\odot 2012$ the authors $\quad 0270-6474 / 12 / 3210587-07 \$ 15.00 / 0$
Studies of flagella and cilia in several different organisms have established the presence of intraflagellar transport (IFT), which involves the anterograde and retrograde movement of proteins, often in complexes known as IFT particles (Kozminski et al., 1993; Cole et al., 1998; Rosenbaum and Witman, 2002; Scholey, 2003; Follit et al., 2009; Hao et al., 2011). IFT is driven by the molecular motors, kinesin-2 in the anterograde direction, and dynein-2 in the retrograde direction (Kozminski et al., 1995; Pazour et al., 1998; Snow et al., 2004; Scholey, 2008).

In photoreceptor cells, genetics studies have shown that opsin mislocalization results from mutant IFT (Pazour et al., 2002; Tsujikawa and Malicki, 2004; Krock and Perkins, 2008; Sukumaran and Perkins, 2009; Keady et al., 2011), and kinesin-2 (Marszalek et al., 2000; Lin-Jones et al., 2003; Jimeno et al., 2006; Avasthi et al., 2009; Insinna et al., 2009; Lopes et al., 2010). Although these studies provide evidence in support of kinesin-2 motor activity in the delivery of opsin along the cilium to the site of disk membrane morphogenesis, opsin mislocalization is not necessarily the result of a direct effect on opsin transport (see Discussion). Recently, it was concluded (Avasthi et al., 2009), in contrast to earlier studies, that the transport of rod opsin (RHO) and other rod outer segment proteins was not dependent on heterotrimeric kinesin-2, suggesting that rod photoreceptors may use a more specialized mechanism to deal with its high volume of traffic.

In the present study we addressed the question of whether the movement of $\mathrm{RHO}$ along the cilium requires heterotrimeric kinesin-2 by studying opsin and IFT in the cilia of live cells, including mouse rod photoreceptor cells. To study the photoreceptor cilia, we developed a method for fluorescence recovery after photobleaching (FRAP) analysis, using retinas of genetically modified mice. This approach has enabled us to test more directly 
the mechanism of movement of the membrane receptor along the most elaborate of primary cilia.

\section{Materials and Methods}

Cell culture. hTERT-RPE1 cells were grown in DMEM/F12 culture medium with $10 \%$ fetal bovine serum (FBS) and 1\% penicillin/streptomycin, at $37^{\circ} \mathrm{C}$ with $5 \% \mathrm{CO}_{2}$. To induce the growth of cilia in confluent cultures, the complete medium was replaced with the medium containing $0.5 \%$ FBS, one day after transfection. They were kept in the low serum condition for $24-48 \mathrm{~h}$ before imaging. Transfection was achieved using Lipofectamine 2000 according to the manufacturer's protocol (Invitrogen). After 3-4 h, normal growth medium was replaced.

Constructs. The Rho-EGFP construct consisted of bovine Rho cDNA, tagged with EGFP at the C terminus, with the C-terminal 24 bp of bovine Rho added to the end of EGFP (Jin et al., 2003). This construct was cloned into a PCDNA3.1 vector that uses the CMV promoter to drive expression. Arl13b-mCherry and Arl13b-CFP were subcloned from mouse Arl13b-GFP, using pN1mCherry and pN1-CFP vectors, respectively, and Kpn1 restriction sites. They were sequenced to check for correct orientation. shRNA constructs against human KIF3A were obtained from Origene (catalog \#TF311911). Human KIF3A\#1 was construct number FI347638 (sequence TGGAAT GAAGACATAGGAGAATGGCAGCT), human KIF3A\#2 was construct number FI347637 (sequence TGCTACAAACAGGCTGTCAGTGTG GATGA), and scrambled control (catalog \#TR30015; sequence GCACTACCAGAGCTAA CTCAGATAGTACT).

FRAP protocol. After making the prebleach fluorescence measurement for $10 \mathrm{~s}$, photobleaching was achieved with repeated full power pulses of a $440 \mathrm{~nm}$ laser for $2 \mathrm{~s}$. Recovery was measured every $0.5 \mathrm{~s}$ for $180 \mathrm{~s}$. Although only rarely observed, cilia that moved out of the plane of focus were disregarded. The images were processed using Volocity software (PerkinElmer). Because there is additional photobleaching that occurs as measurements are made during recovery, the plotted fluorescence recovery was normalized to the prebleach fluorescence level. A single exponential fit was made for the recording from each cilium, and the time taken to reach half of the fluorescence recovery $\left(t_{1 / 2}\right)$ was determined from this curve. Statistical analyses were performed using the Student's $t$ test. All error bars indicate \pm SEM.

Mouse breeding. Animal use followed National Institutes of Health guidelines under an approved protocol. Mice were kept on a $12 \mathrm{~h} \mathrm{light/}$ dark cycle under 10-50 lux of fluorescent lighting during the light cycle. The genetic mutants used were as follows: Kif $3 a^{\text {flox/flox }}$ (Marszalek et al., 2000), RHO-Cre (line 8) transgenic (Jimeno et al., 2006), Rpe65 knockout (Redmond et al., 1998), and RHO-EGFP knock-in (Chan et al., 2004). RHO-Cre ${ }^{+}$mice always contained only one transgenic allele. RHO-EGFP mice were crossed with Rpe65 $5^{-1-}$ to obtain mice that were heterozygous for RHO-EGFP and homozygous for Rpe65 null. Breeders were checked every day for the birth of a litter during the previous night; the day a litter was first observed was regarded as postnatal day 0 (P0).

Retinal explants. Eyes from mice of either gender were enucleated and immediately put in Neurobasal medium (Invitrogen) under room light. The cornea and lens were removed and the resulting eyecup was cut into small pieces. These small pieces were transferred in medium to the imaging chamber (glass bottom, $0.1 \mathrm{~mm}$ thick). The chamber was closed and sealed with nail polish. These explants were transferred to the imaging incubator and imaged at $37^{\circ} \mathrm{C}$. Rod photoreceptors lying parallel to the coverglass (usually at the edges of the explant) were used for the FRAP protocol.

Microscopy and immunocytochemistry. Live microscopy and FRAP were performed using a spinning disk confocal microscope (PerkinElmer) or an FV1000 Olympus scanning laser confocal microscope. The latter was also used for imaging cells that were fixed in $4 \%$ buffered formaldehyde, and labeled with antibodies against KIF17 (catalog \#ab11261; Abcam), acetylated $\alpha$-tubulin (catalog \#T7451; Sigma), or KAP3 (catalog \#SC-8877; Santa Cruz Biotechnology).

Western blot analysis. Western blots to assess the efficacy of shRNA constructs against human KIF3A were performed with 293T and hTERTRPE1 cell lysates. Cells were transfected with shRNA constructs, including a scrambled control, in 6-well plates at $50-60 \%$ confluence. The medium was changed $4-5 \mathrm{~h}$ after transfection and cells were kept at $37^{\circ} \mathrm{C}$, $5 \% \mathrm{CO}_{2}$ for $48 \mathrm{~h}$. Three micrograms or $10 \mu \mathrm{g}$ of total protein were loaded per well for SDS-PAGE. The membranes were labeled with KIF3A (catalog \#ab11259;Abcam) and glyceraldehyde-3-phosphate dehydrogenase (catalog \#ab9484; Abcam) antibodies.

\section{Results}

Opsin in the cilia of transfected epithelial cells

We chose to study hTERT-RPE1 cells, in part because they have a cilium that protrudes from a pocket in the apical membrane, not unlike that of the photoreceptor cilium (Peters et al., 1983; MollaHerman et al., 2010). Cells were transfected with a construct encoding a RHO-EGFP fusion protein of RHO with a C-terminal 

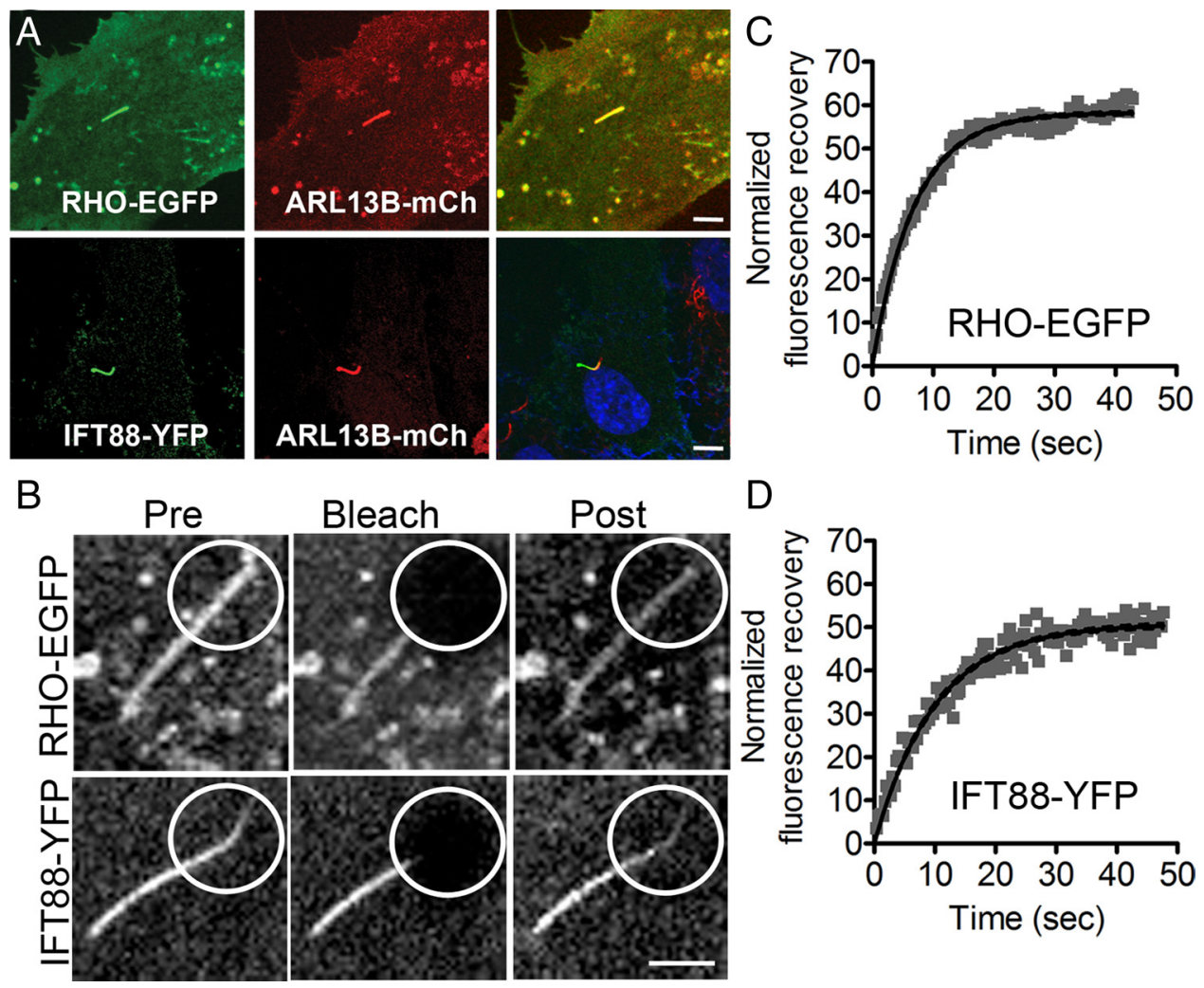

Figure 2. FRAP of RH0 and IFT88 in cilia of hTERT-RPE1 cells. A, Cells cotransfected to express either RH0-EGFP with ARL13B-mCherry or IFT88-YFP with ARL13B-mCherry. ARL13B-mCherry was used as a live-cell cilium marker. Right, Shows overlays. Scale bar, $5 \mu \mathrm{m}$. B, Images illustrating fluorescence recovery of RHO-EGFP and IFT88-YFP in the distal cilium. Pre, Prebleach; Post, recovery $3 \mathrm{~min}$ after photobleaching. Scale bar, $1 \mu \mathrm{m}$. C, D, Examples of plots of normalized fluorescence recovery in the distal cilium vs time.

EGFP, similar to that shown previously to be targeted to the outer segment and to function normally (Jin et al., 2003). In a prior study, opsin was found to be targeted to the apical membrane of transfected Madin-Darby canine kidney cells (Chuang and Sung, 1998). We observed that RHO-EGFP was also targeted apically in hTERT-RPE1 cells. Upon further inspection, we observed that a relatively high concentration of the EGFP signal colocalized with acetylated $\alpha$-tubulin in the cilium (Fig. $1 A$ ). IFT88-YFP was similarly localized (Fig. $1 B$ ), and antibodies against KAP3 (the nonmotor subunit of heterotrimeric kinesin-2) and KIF17 (a homodimeric kinesin-2) indicated that these two kinesin-2 motors are endogenously expressed in hTERT-RPE1 cells and colocalize with acetylated tubulin in the cilium (Fig. 1C,D).

\section{FRAP of RHO-EGFP in the cilium of hTERT-RPE1 cells}

To study the movement of RHO-EGFP in the cilium, we developed a protocol based on FRAP, using hTERT-RPE1 cells. Transfected cells were serum starved to induce the formation of cilia. RHOEGFP and IFT88-YFP were coexpressed with ARL13B-mCherry, which was used as a cilium marker (Hori et al., 2008) (Fig. 2A). IFT88 has been shown to be important for photoreceptor outer segment assembly (Pazour et al., 2002), and a component of kinesin-2based anterograde transport in cilia (Cole et al., 1998; Pazour et al., 2000). The cilia of hTERT-RPE1 cells lie almost parallel to the apical surface, and with their basal bodies closer to the nucleus (MollaHerman et al., 2010), thus allowing for identification of the basal and apical ends. For these experiments, a $\sim 1 \mu \mathrm{m}$ circular region, covering the distal half of the cilium, was bleached, so that any fluorescence recovery must result from movement from the basal part of the cilium. Figure $2 B$ shows examples of recovery of RHO-EGFP and IFT88-YFP fluorescence after photobleaching, respectively. The time taken to reach half of the fluorescence recovery $\left(t_{1 / 2}\right)$, as determined from a single exponential fit of the data, varied somewhat among different cells, but was of a similar order of magnitude for RHO-EGFP and IFT88-YFP, with no significant difference found between the means (Fig. $2 C, D$ ) (mean $t_{1 / 2}$ was $4.1 \pm 0.8 \mathrm{~s}$ for RHO$\operatorname{EGFP}(n=11)$ and $6.0 \pm 0.8 \mathrm{~s}$ for IFT88-YFP $(n=18) ; p=0.1)$.

To test whether heterotrimeric kinesin-2 is required for the ciliary movement of opsin, we transfected hTERT-RPE1 cells with shRNA against human KIF3A, the obligatory motor subunit of this kinesin. To allow identification of cells expressing the shRNA, red fluorescent protein (RFP) was expressed as a reporter from another cistron (Fig. 3A). First, we assessed the efficacy of the shRNA constructs in $293 \mathrm{~T}$ cells, since only a minority of hTERT-RPE1 cells are typically transfected. The RFP reporter indicated variation from cell to cell, but on average, by Western blot analysis, two forms of shRNA were found to reduce KIF3A levels, compared with a scrambled shRNA, with shRNA\#2 proving to be more efficacious than shRNA\#1, with a $50 \%$ reduction in KIF3A. In cultures of hTERT-RPE1 cells, with 30\% of the cells transfected with the shRNA\#2, total KIF3A in the culture was reduced by $\sim 20 \%$, as measured by Western blot analysis (Fig. $3 B$ ).

hTERT-RPE1 cells were cotransfected with Rho-EGFP, Arl13b$C F P$, and one of the shRNA constructs. Two days after transfection, the cells were moved to culture medium containing low serum for $\sim 24 \mathrm{~h}$ to induce ciliogenesis. FRAP was performed on cilia of cells expressing high levels of RFP (Fig. $3 A$ ). However, in the cultures treated with shRNA\#2 there were fewer long cilia and more cell death than observed in control cultures. Therefore, to minimize recording from degenerate cilia, we selected cilia that were at least $1.5 \mu \mathrm{m}$ long. The mean length of cilia from which measurements were obtained was $3.0 \mu \mathrm{m}$ for the shRNA\#1, $2.7 \mu \mathrm{m}$ for the shRNA\#2, and $2.9 \mu \mathrm{m}$ 
for the scrambled control. The mean $t_{1 / 2}$ measured from cells with the shRNA\#2 was increased by $\sim 2$-fold over that from cells expressing the scrambled control, while no significant difference was observed with shRNA\#1 (Fig. 3C,D).

\section{FRAP of RHO-EGFP in the connecting} cilium of rod photoreceptor cells

Next, we used FRAP to analyze the movement of opsin in the connecting cilium of mouse rod photoreceptor cells. The photoreceptor cilium extends from a partly enclosed pocket on the side of the inner segment. Traditionally, the region between the basal body and disk membranes ( $\sim 1.5 \mu \mathrm{m}$ in length) has been termed the connecting cilium, since it appears to connect the inner and outer segments. The connecting cilium is the structural equivalent of the cilium and flagellum transition zone (Röhlich, 1975; Besharse and Horst, 1990; Insinna and Besharse, 2008). For these studies, we used isolated retinas from mice that were heterozygous for the knock-in of a human RHO gene fused to EGFP in place of the mouse RHO gene (Fig. 4A). The RHO-EGFP in these mice is expressed and targeted to the outer segment like that of the wild-type opsin (Chan et al., 2004). A problem with using these mice for live cell imaging is that the wavelength of light that excites EGFP coincides with that for RHO activation, so that activation of the EGFP leads to excessive phototransduction and poor photoreceptor cell survival in retinal explants. To obviate this problem, we generated $R H O-E G F P$ mice that also lacked a functional Rpe65 gene by crossing them with Rpe65 $5^{-1-}$ mice. Rpe65 $5^{-1-}$ mice lack the isomerase required to regenerate the rhodopsin (Redmond et al., 1998), and so are insensitive to the laser used to excite the RHO-EGFP. However, opsin is still transported to the rod outer segments in Rpe65 ${ }^{-1-}$ mice (Redmond et al., 1998). Our studies were performed on P9-P12 mice that were heterozygous for $\mathrm{RHO}$ EGFP; the lack of RPE65 in these mice had no effect on the localization of RHO-EGFP (Fig. 4A-C).

FRAP analysis was performed on rod photoreceptors that were lying parallel to the coverglass (usually at the edges of the explant). We experimented with preparations that were either pieces of eyecup or pieces of sensory retina, and found that the fluorescence recovery rates were essentially the same in both cases. Recovery of RHO-EGFP fluorescence was observed when a region corresponding to the location of the connecting cilium (Fig. $4 B$, arrow) was bleached (Fig. 4D,E). When the bleaching spot included the outer segment, even only a small part together with connecting cilium, no detectable recovery was recorded over $3 \mathrm{~min}$. Figure $4, F$ and $G$, shows the result of a FRAP recording from the distal outer segment of the same cell as that shown in Figure 4D. The outer segment disks are a much larger source of RHO-EGFP than the cilium, both in terms of volume and concentration; however, their fluorescence recovery is likely dependent on their distal displacement, which takes place over a longer time period (Young, 1967).

To study the effect of KIF3A depletion on the movement of ciliary opsin in rod photoreceptors, we used P12 mice that we bred to contain RHO-Cre;Kif $3 a^{\text {flox/flox }}$ in addition to Rpe65 $5^{-1-}$ and RHO-EGFP. The RHO-Cre that was used has been shown previously to provide synchronous expression of Cre and uniform excision of $\mathrm{Kif} \mathrm{a}^{\text {flox }}$ in rod photoreceptors, shortly after ciliogenesis. In the absence of a floxed gene, this RHO-Cre trans-
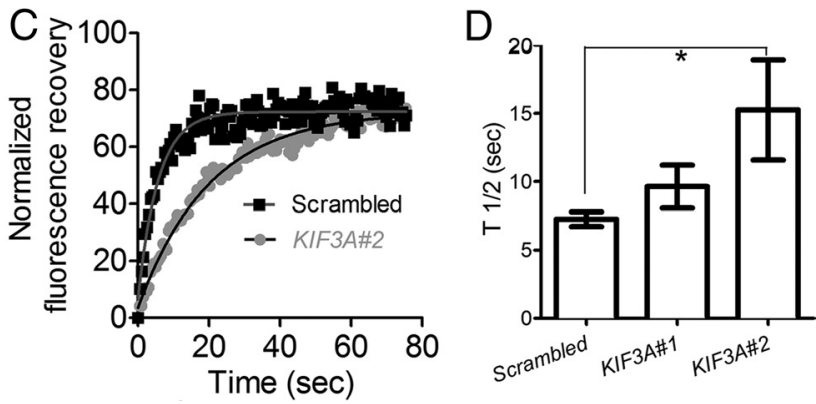

Figure 3. Reduction in FRAP of RHO-EGFP in the cilium of hTERT-RPE1 cells, following knock down of KIF3A. $A$, Fluorescence image of a scrambled contol ShPNA, probed recovery versus time for $\mathrm{RHO}$-EGFP in the distal cilium of cells expressing KIF3A\#2 or scrambled ShRNA. $D$ Bar graph of the measured half-times, $t_{1 / 2}$, for recovery of RHO-EGFP in the presence of wild-type or reduced levels of KIF3A ( $n=27$ for scrambled; $n=21$ for $K I F 3 A \# 1$; $n=27$ for KIF3A\#2; $p<0.01$ for scrambled vs KIF3A\#2, indicated by asterisk).

gene results in photoreceptor degeneration that is evident by $\mathrm{P} 42$; however, by this stage, the level of CRE is an order of magnitude higher than at P12. In P12 RHO-Cre;Kif $3 a^{\text {flox/flox }}$ mice, the level of KIF3A in the rod photoreceptors is reduced to less than half, and photoreceptor cell degeneration, which begins in earnest after the second week, is still negligible (Jimeno et al., 2006).

FRAP analysis showed that the movement of RHO-EGFP in connecting cilia of KIF3A-deficient rod photoreceptors was $\sim 4$ fold less than that in controls, which included Kif $3 a^{+/+}$mice with or without the RHO-Cre transgene (Fig. 5).

\section{Discussion}

The localization of membrane receptors or channels in the plasma membrane of primary cilia is crucial to the functioning of these cilia in detecting extracellular signals. Previous reports have proposed that kinesin-2 in conjunction with IFT proteins transport ciliary membrane proteins from the base of the cilium along the ciliary plasma membrane. However, direct evidence to support this hypothesis is limited. Here, we focused on the visual receptor, opsin, which is transported in the most elaborate of all cilia, the outer segment of photoreceptor cells. Previous studies have focused on retrospective analyses of mutant retinas, and have resulted in conflicting conclusions. Here, we have taken a more direct approach, using FRAP analysis of live cells expressing fluorescently tagged opsin. These FRAP data indicate that the movement of ciliary opsin is comparable to that of one of the IFT proteins, IFT88, and is dependent on heterotrimeric kinesin-2, in both photoreceptor cells and cells of an epithelial cell line.

Heterotrimeric kinesin-2 was first identified in sea urchin eggs (Cole et al., 1993). It was then found to be necessary for the formation of a variety of cilia and flagella, and IFT movements along cilia and flagella (Kozminski et al., 1995; Morris and Scholey, 1997; Piperno and Mead, 1997; Cole et al., 1998). Although none of the IFT proteins appears to be a transmembrane protein (Cole, 2003), there is some evidence that membrane proteins may be linked to IFT and thus moved along the ciliary plasma membrane. Polystyrene beads applied to the surface of Chlamydomonas flagella have been observed moving along them (Kozminski et al., 1993). The movement of one type of channel in Caenorhabditis elegans sensory neurons was found to be comparable to that of IFT proteins [although motility of another channel was not detected (Qin et al., 2005)]. Finally, genetic evidence has shown that IFT is required for the normal localization of ciliary membrane proteins, such as polycystins (Follit et al., 2006), 

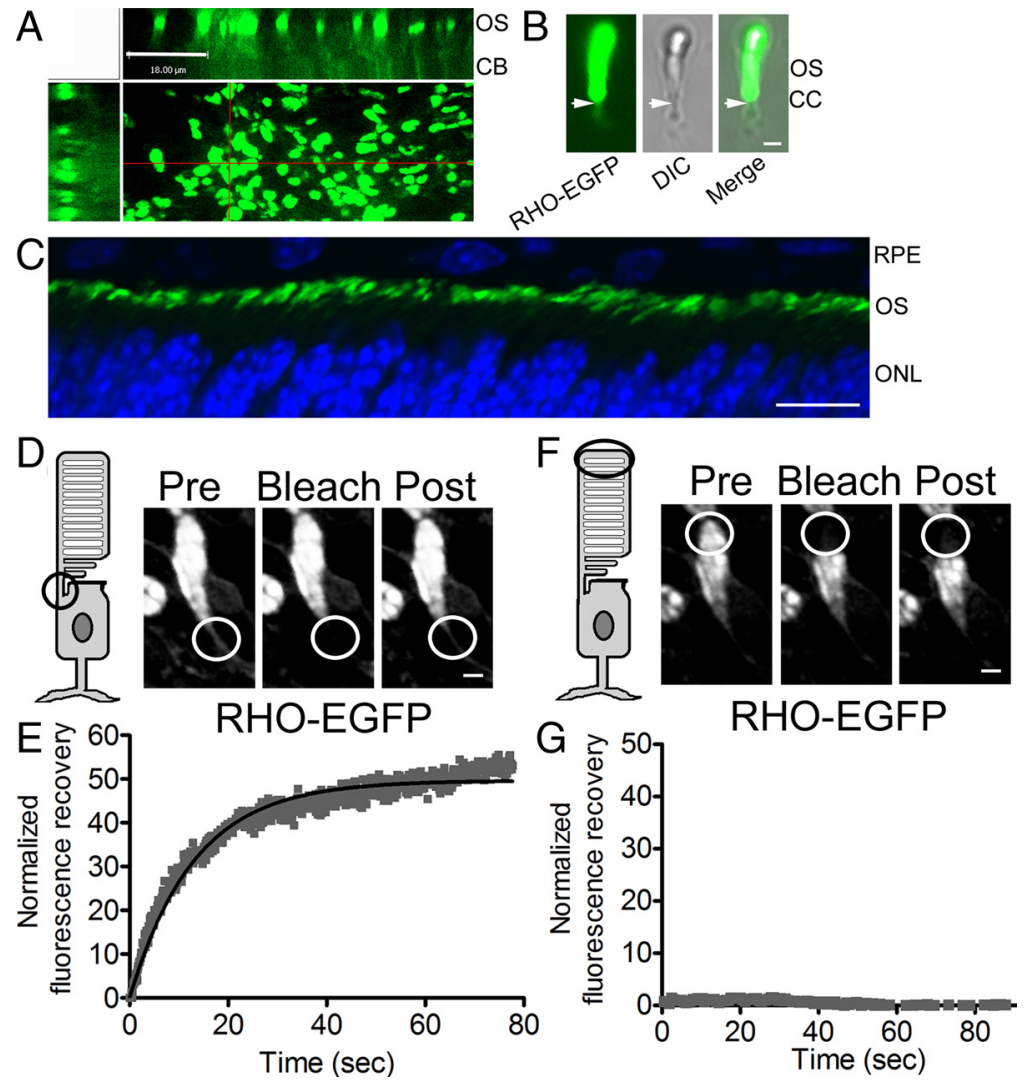

Figure 4. FRAP of RHO-EGFP in cilia of mouse rod photoreceptor cells. $A$, Fluorescence micrograph of a retinal explant from a P10 RHO-EGFP;Rpe65 ${ }^{-1-}$ mouse. The main image views the retina as a flatmount (looking down on the fluorescent rod outer segments; ROSs). The other images showz-axis reconstructions along the regions indicated by the red lines. $0 S$, outer segments; $C B$, cell body region. Scale bar, $18 \mu \mathrm{m}$. B, Fluorescence and DIC images of a single rod photoreceptor cell in a retinal explant from a P13 RHO-EGFP mouse. The arrows indicate the region immediately below the base of the 0 S. This region is the location of the connecting cilium $(C \mathrm{C})$ and was targeted for FRAP studies of RHO-EGFP. Scale bar, $1 \mu \mathrm{m}$. C, Fluorescence image of a cryosection of retina of a P10 RHO-EGFP;Rpe65 ${ }^{-1-}$ mouse, showing RHO-EGFP (green) and DAPI staining of nuclei (blue). Scale bar, $10 \mu \mathrm{m}$. RPE, retinal pigment epithelium; ONL, outer nuclear layer. D-G, FRAP using retinal explants from RHO-EGFP;Rpe65 ${ }^{-1-}$ mice. D, F, FRAP of two different locations of a rod photoreceptor: the CC (D) and the distal region of the ROS $(\boldsymbol{F}$ ). Images illustrate RHO-EGFP before the photobleach (Pre), and immediately after (Bleach) or 3 min after (Post). Scale bars: (in $\boldsymbol{D}, \boldsymbol{F}), 3 \mu \mathrm{m}$. E, G, Plots of normalized fluorescence recovery versus time. FRAP experiments were performed on $42 \mathrm{rod}$ photoreceptor cells from 10 different RHO-EGFP;Rpe65 ${ }^{-1-}$ retinas.
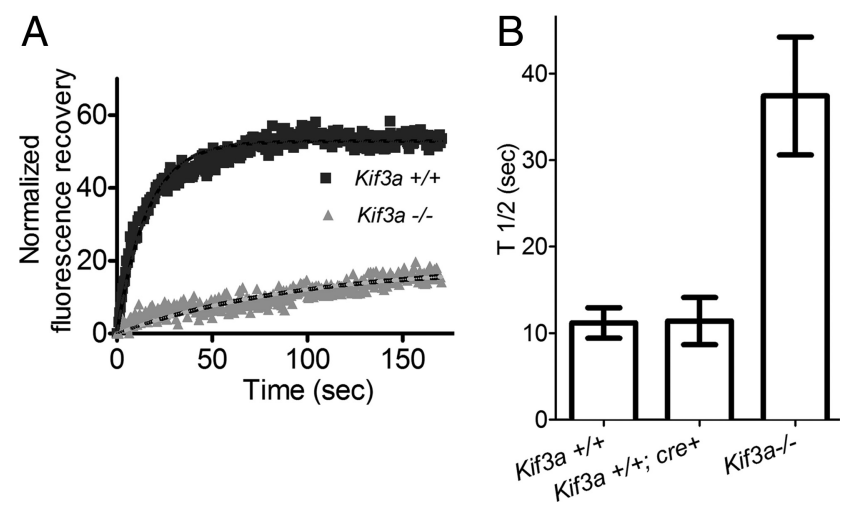

Figure 5. Reduction in FRAP of RHO-EGFP in the connecting cilium of $\mathrm{Kif3a}^{-1-}$ mouse rod photoreceptor cells. $A$, Example of plot of normalized fluorescence recovery versus time for RHO-EGFP in the connecting cilia of $\mathrm{Kif3}^{+/+}$and $\mathrm{Kif3}^{-/-}$rod photoreceptors (full genotypes: RHO-EGFP(KI);

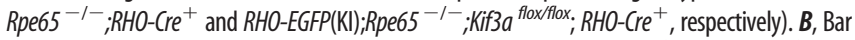
graph of the measured half-times, $t_{1 / 2}$, for recovery of RHO-EGFP in $\mathrm{Kif3a}^{+/+}$and $\mathrm{Kif3}^{-/-}$rod photoreceptors. $\mathrm{Kif3a}^{+/+} ; \mathrm{Cre}^{+}$represents RHO-EGFP(K));Rpe65 ${ }^{-1-}$; $\mathrm{RHO}-\mathrm{Cre}{ }^{+}$(i.e., lacking the floxed gene, but with the (re transgene). Measurements of $t_{1 / 2}$ were $11.2 \pm 1.7,11.4 \pm 2.7$, and $37.4 \pm 6.8 \mathrm{~s}$, for Kif3a ${ }^{+/+}(n=42), K_{i f 3 a^{+/+}} ; \mathrm{Cre}^{+}(n=10)$, and Kif3a ${ }^{-/-}(n=24)$, respectively $\left(p<0.0001\right.$ for Kif3a $a^{+/+}$vs Kif3a ${ }^{-/-}, p<0.01$ for Kif3a ${ }^{+/+}$; Cre $^{+}$vs Kif3a ${ }^{-/-}, p=0.96$ for $\mathrm{Kif3a}^{+/+}$vs Kif3a $\left.{ }^{+/+} ; \mathrm{Cre}^{+}\right)$. the olfactory cyclic nucleotide-gated channel (Jenkins et al., 2006), and smoothened (Corbit et al., 2005).

Immunodetection of KIF3A (Beech et al., 1996; Muresan et al., 1997; Whitehead et al., 1999) and IFT proteins (Pazour et al., 2002; Tsujikawa and Malicki, 2004; Krock and Perkins, 2008; Luby-Phelps et al., 2008; Sedmak and Wolfrum, 2010) in the connecting cilium of photoreceptor cells suggest that IFT driven by heterotrimeric kinesin-2 might have a transport function in this very specialized cilium as well. Support for a role in the transport of opsin was obtained in a Cre-loxP mutagenesis study, in which the Kif3a gene was deleted in mature mouse photoreceptor cells, using an IRBP-Cre transgene in combination with Kif $3 a^{f l o x} f f l o x$. The resulting loss of heterotrimeric kinesin-2 function caused opsin mislocalization and photoreceptor cell death (Marszalek et al., 2000). Use of a $\mathrm{RHO}-\mathrm{Cre}$ transgene that was expressed robustly and uniformly across the entire rod photoreceptor population demonstrated more clearly the temporal relationship among Cre expression, loss of KIF3A, mislocalization of opsin, and photoreceptor cell death, thus adding further support to such a role (Jimeno et al., 2006). However, more recently, Avasthi et al. (2009), using a different Rho-

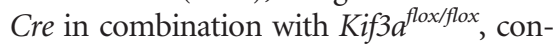
cluded that the presence of opsin in the outer segments of mutant retinas indicated KIF3A was not required for opsin delivery to the outer segment, even though some opsin mislocalization was observed.

There are two problems with using opsin mislocalization in fixed tissue as an indicator of a direct effect on ciliary opsin transport. First, opsin mislocalization is not unique to trafficking defects. It is also a characteristic of photoreceptor cells in a variety of retinal degeneration models that appear to be unrelated to defects in opsin trafficking, such as the $r d$ and $r d s$ mice, and the RCS rat (Nir and Papermaster, 1986; Jansen et al., 1987; Nir et al., 1987, 1989; Usukura and Bok, 1987). Second, IFT and heterotrimeric kinesin-2 are required for ciliogenesis (Lin et al., 2003; Fan et al., 2004). Hence, to test for a role in opsin delivery along the connecting cilium to the disk membranes, the knock down of IFT protein or kinesin motor function must be incomplete (Pazour et al., 2002) or occur after the connecting cilium has formed (Marszalek et al., 2000; Jimeno et al., 2006; Avasthi et al., 2009). Thus some opsin is still trafficked to the outer segment, at least initially (Besharse et al., 1985). In the previous gene knock-down studies, the results were less clear due to lack of discrimination between the opsin synthesized before and after the knock down. With FRAP analysis, we were able to observe and measure opsin movement along the cilium in live cells. The twofold or fourfold decrease in rate of movement (the reciprocal of the $t_{1 / 2}$ of recovery) that was measured in KIF3Adeficient hTERT-RPE1 or photoreceptor cells, respectively, thus provides a more direct indication of a role for heterotrimeric kinesin-2 function in ciliary opsin transport. 
A requirement for heterotrimeric kinesin-2 in ciliary opsin transport could be effected by this motor and associated IFT proteins directly moving opsin molecules along the membrane as connected cargo. Alternatively, the motor activity could set up a bulk flow of membrane components, in which opsin gets carried along. Or, third, heterotrimeric kinesin- 2 may be required only for ciliary maintenance, without which a more specialized opsin motor is unable to function. Arguing against this last suggestion, electron microscopic studies show that the connecting cilia of rod photoreceptors appear normal in RHO-Cre; Kif $3 a^{f l o x / f l o x}$ mice of a similar age to those used in the present study (Jimeno et al., 2006). Moreover, the observation of opsin movement in the less specialized primary cilium of hTERTRPE1 cells suggests that a common motor, such as kinesin-2/IFT, can support the movement, and that a specialized photoreceptor cilium motor seems to be unnecessary for opsin transport.

The present result showing that the movement of ciliary opsin was comparable to that of one of the IFT proteins, IFT88, suggests that opsin and kinesin/IFT may move together. Similar rates of movement could also be a feature of a bulk flow mechanism. On the other hand, a previous report that opsin coimmunoprecipitates with IFT88 from retinal extracts (Bhowmick et al., 2009) supports the notion that opsin may be a specific cargo of kinesin2/IFT. The rate of opsin movement that we measured by FRAP of $\sim 1 \mu \mathrm{m}$ length of connecting cilium $\left(t_{1 / 2} \sim 10 \mathrm{~s}\right)$ is quite high, but should be within the capabilities of a motor that travels $\sim 0.7$ $\mu \mathrm{m} / \mathrm{s}$, as shown for kinesin-2 along the doublet microtubules of a primary cilium (Snow et al., 2004).

FRAP of RHO-EGFP could potentially be effected by diffusion; however, in this case, the recovery might be expected to be faster. Measurements of FRAP by diffusion for a variety of proteins suggest that a transmembrane protein that is comparable to RHO-EGFP would diffuse at a rate that is $\sim 2$ orders of magnitude faster than the movement measured in the present experiments, so that any diffusion would contribute only to the very first point of the FRAP curve (Sprague and McNally, 2005). An estimated $t_{1 / 2}$ for RHO, based on the diffusion coefficient measured for RHO in disk membranes (Wey et al., 1981), would be $\sim 1$ order of magnitude faster than that measured here. Since diffusion should be unaffected by depressed levels of KIF3A, our observation of FRAP inhibition under this condition provides evidence against a role for diffusion, either in a distal direction or in a retrograde diffusion from the disk membranes. Indeed, this result supports the presence of a diffusion barrier in the connecting cilium for preventing the return of $\mathrm{RHO}$ from the outer segment.

Opsin is continually delivered to the base of the photoreceptor cilium, and then transported along the transition zone (a.k.a. connecting cilium), to support the renewal of the phototransductive disk membranes, throughout the life of the organism (Young, 1967, 1968; Papermaster et al., 1985; Deretic and Papermaster, 1991). The demand placed on the transport of opsin by new disk membrane formation is high. In mouse rod photoreceptors, $\sim 2000$ molecules of opsin per minute are required (Besharse and Horst, 1990), and in frog rod photoreceptors, $\sim 50,000$ opsin molecules per minute are required (Besharse et al., 1977; Papermaster et al., 1985). The connecting cilium is also the conduit for fluxes of cytosolic proteins, such as arrestin, transducin, and recoverin, which translocate between the inner and outer segments in response to changes in light intensity (Calvert et al., 2006). Despite this extraordinary trafficking activity, the present study supports a role for the heterotrimeric kinesin-2/IFT system that is conserved across all cilia and flagella. Other conserved proteins also function in the photoreceptor cilium, such as the homodimeric kinesin-2, KIF17 (Ou et al., 2005; Pan et al., 2006). A role for KIF17 has been demonstrated in zebrafish cone photoreceptors (Insinna et al., 2009). More generally, many of the ciliopathies, caused by mutations in genes encoding various cilium proteins, also include photoreceptor degeneration (Badano et al., 2005; Hildebrandt et al., 2011), thus emphasizing the conserved nature of the photoreceptor cilium.

In conclusion, we have observed and measured the movement of opsin in the cilia of an epithelial cell line and those of photoreceptor cells. The opsin movement was comparable to that of IFT88 and impaired when KIF3A was depleted. The present study therefore provides evidence from live cell analysis that the conserved heterotrimeric kinesin-2/IFT motor system is required for the transport of opsin along the ciliary plasma membrane.

\section{References}

Avasthi P, Watt CB, Williams DS, Le YZ, Li S, Chen CK, Marc RE, Frederick JM, Baehr W (2009) Trafficking of membrane proteins to cone but not rod outer segments is dependent on heterotrimeric kinesin-II. J Neurosci 29:14287-14298.

Badano JL, Teslovich TM, Katsanis N (2005) The centrosome in human genetic disease. Nat Rev Genet 6:194-205.

Beech PL, Pagh-Roehl K, Noda Y, Hirokawa N, Burnside B, Rosenbaum JL (1996) Localization of kinesin superfamily proteins to the connecting cilium of fish photoreceptors. J Cell Sci 109:889-897.

Besharse JC, Horst CJ (1990) The photoreceptor connecting cilium: a model for the transition zone. In: Ciliary and flagellar membranes (Bloodgood RA, ed), pp 409-431. New York: Plenum.

Besharse JC, Hollyfield JG, Rayborn ME (1977) Turnover of rod photoreceptor outer segments. II. Membrane addition and loss in relationship to light. J Cell Biol 75:507-527.

Besharse JC, Forestner DM, Defoe DM (1985) Membrane assembly in retinal photoreceptors. III. Distinct membrane domains of the connecting cilium of developing rods. J Neurosci 5:1035-1048.

Bhowmick R, Li M, Sun J, Baker SA, Insinna C, Besharse JC (2009) Photoreceptor IFT complexes containing chaperones, guanylyl cyclase 1 and rhodopsin. Traffic 10:648-663.

Calvert PD, Strissel KJ, Schiesser WE, Pugh EN Jr, Arshavsky VY (2006) Light-driven translocation of signaling proteins in vertebrate photoreceptors. Trends Cell Biol 16:560-568.

Chan F, Bradley A, Wensel TG, Wilson JH (2004) Knock-in human rhodopsin-GFP fusions as mouse models for human disease and targets for gene therapy. Proc Natl Acad Sci U S A 101:9109-9114.

Chuang JZ, Sung CH (1998) The cytoplasmic tail of rhodopsin acts as a novel apical sorting signal in polarized MDCK cells. J Cell Biol 142:1245-1256.

Cole DG (2003) The intraflagellar transport machinery of Chlamydomonas reinhardtii. Traffic 4:435-442.

Cole DG, Chinn SW, Wedaman KP, Hall K, Vuong T, Scholey JM (1993) Novel heterotrimeric kinesin-related protein purified from sea urchin eggs. Nature 366:268-270.

Cole DG, Diener DR, Himelblau AL, Beech PL, Fuster JC, Rosenbaum JL (1998) Chlamydomonas kinesin-II-dependent intraflagellar transport (IFT): IFT particles contain proteins required for ciliary assembly in Caenorhabditis elegans sensory neurons. J Cell Biol 141:993-1008.

Corbit KC, Aanstad P, Singla V, Norman AR, Stainier DY, Reiter JF (2005) Vertebrate Smoothened functions at the primary cilium. Nature 437:1018-1021.

Deretic D, Papermaster DS (1991) Polarized sorting of rhodopsin on postGolgi membranes in frog retinal photoreceptor cells. J Cell Biol 113:1281-1293.

Fan S, Hurd TW, Liu CJ, Straight SW, Weimbs T, Hurd EA, Domino SE, Margolis B (2004) Polarity proteins control ciliogenesis via kinesin motor interactions. Curr Biol 14:1451-1461.

Follit JA, Tuft RA, Fogarty KE, Pazour GJ (2006) The intraflagellar transport protein IFT20 is associated with the Golgi complex and is required for cilia assembly. Mol Biol Cell 17:3781-3792.

Follit JA, Xu F, Keady BT, Pazour GJ (2009) Characterization of mouse IFT complex B. Cell Motil Cytoskeleton 66:457-468.

Hao L, Thein M, Brust-Mascher I, Civelekoglu-Scholey G, Lu Y, Acar S, Prevo B, Shaham S, Scholey JM (2011) Intraflagellar transport delivers tubulin isotypes to sensory cilium middle and distal segments. Nat Cell Biol 13:790-798. 
Hildebrandt F, Benzing T, Katsanis N (2011) Ciliopathies. N Engl J Med 364:1533-1543.

Hori Y, Kobayashi T, Kikko Y, Kontani K, Katada T (2008) Domain architecture of the atypical Arf-family GTPase Arl13b involved in cilia formation. Biochem Biophys Res Commun 373:119-124.

Insinna C, Besharse JC (2008) Intraflagellar transport and the sensory outer segment of vertebrate photoreceptors. Dev Dyn 237:1982-1992.

Insinna C, Humby M, Sedmak T, Wolfrum U, Besharse JC (2009) Different roles for KIF17 and kinesin II in photoreceptor development and maintenance. Dev Dyn 238:2211-2222.

Jansen HG, Sanyal S, De Grip WJ, Schalken JJ (1987) Development and degeneration of retina in rds mutant mice: ultraimmunohistochemical localization of opsin. Exp Eye Res 44:347-361.

Jenkins PM, Hurd TW, Zhang L, McEwen DP, Brown RL, Margolis B, Verhey KJ, Martens JR (2006) Ciliary targeting of olfactory CNG channels requires the CNGB1b subunit and the kinesin-2 motor protein, KIF17. Curr Biol 16:1211-1216.

Jimeno D, Feiner L, Lillo C, Teofilo K, Goldstein LS, Pierce EA, Williams DS (2006) Analysis of kinesin-2 function in photoreceptor cells using synchronous Cre-loxP knock-out of Kif $3 a$ with $\mathrm{RHO}$-Cre. Invest Ophthalmol Vis Sci 47:5039-5046.

Jin S, McKee TD, Oprian DD (2003) An improved rhodopsin/EGFP fusion protein for use in the generation of transgenic Xenopus laevis. FEBS Lett 542:142-146.

Keady BT, Le YZ, Pazour GJ (2011) IFT20 is required for opsin trafficking and photoreceptor outer segment development. Mol Biol Cell 22:921-930.

Kozminski KG, Johnson KA, Forscher P, Rosenbaum JL (1993) A motility in the eukaryotic flagellum unrelated to flagellar beating. Proc Natl Acad Sci U S A 90:5519-5523.

Kozminski KG, Beech PL, Rosenbaum JL (1995) The Chlamydomonas kinesin-like protein FLA10 is involved in motility associated with the flagellar membrane. J Cell Biol 131:1517-1527.

Krock BL, Perkins BD (2008) The intraflagellar transport protein IFT57 is required for cilia maintenance and regulates IFT-particle-kinesin-II dissociation in vertebrate photoreceptors. J Cell Sci 121:1907-1915.

Lin F, Hiesberger T, Cordes K, Sinclair AM, Goldstein LS, Somlo S, Igarashi P (2003) Kidney-specific inactivation of the KIF3A subunit of kinesin-II inhibits renal ciliogenesis and produces polycystic kidney disease. Proc Natl Acad Sci U S A 100:5286-5291.

Lin-Jones J, Parker E, Wu M, Knox BE, Burnside B (2003) Disruption of kinesin II function using a dominant negative-acting transgene in Xenopus laevis rods results in photoreceptor degeneration. Invest Ophthalmol Vis Sci 44:3614-3621.

Lopes VS, Jimeno D, Khanobdee K, Song X, Chen B, Nusinowitz S, Williams DS (2010) Dysfunction of heterotrimeric kinesin-2 in rod photoreceptor cells and the role of opsin mislocalization in rapid cell death. Mol Biol Cell 23:4076-4088.

Luby-Phelps K, Fogerty J, Baker SA, Pazour GJ, Besharse JC (2008) Spatial distribution of intraflagellar transport proteins in vertebrate photoreceptors. Vision Res 48:413-423.

Marszalek JR, Liu X, Roberts EA, Chui D, Marth JD, Williams DS, Goldstein LS (2000) Genetic evidence for selective transport of opsin and arrestin by kinesin-II in mammalian photoreceptors. Cell 102:175-187.

Molla-Herman A, Ghossoub R, Blisnick T, Meunier A, Serres C, Silbermann F, Emmerson C, Romeo K, Bourdoncle P, Schmitt A, Saunier S, Spassky N, Bastin P, Benmerah A (2010) The ciliary pocket: an endocytic membrane domain at the base of primary and motile cilia. J Cell Sci 123:1785-1795.

Morris RL, Scholey JM (1997) Heterotrimeric kinesin-II is required for the assembly of motile $9+2$ ciliary axonemes on sea urchin embryos. J Cell Biol 138:1009-1022.

Muresan V, Bendala-Tufanisco E, Hollander BA, Besharse JC (1997) Evidence for kinesin-related proteins associated with the axoneme of retinal photoreceptors. Exp Eye Res 64:895-903.

Nir I, Papermaster DS (1986) Immunocytochemical localization of opsin in the inner segment and ciliary plasma membrane of photoreceptors in retinas of rds mutant mice. Invest Ophthalmol Vis Sci 27:836-840.

Nir I, Sagie G, Papermaster DS (1987) Opsin accumulation in photoreceptor inner segment plasma membranes of dystrophic RCS rats. Invest Ophthalmol Vis Sci 28:62-69.

Nir I, Agarwal N, Sagie G, Papermaster DS (1989) Opsin distribution and synthesis in degenerating photoreceptors of rd mutant mice. Exp Eye Res 49:403-421.

Ou G, Blacque OE, Snow JJ, Leroux MR, Scholey JM (2005) Functional coordination of intraflagellar transport motors. Nature 436:583-587.

Pan X, Ou G, Civelekoglu-Scholey G, Blacque OE, Endres NF, Tao L, Mogilner A, Leroux MR, Vale RD, Scholey JM (2006) Mechanism of transport of IFT particles in C. elegans cilia by the concerted action of kinesin-II and OSM-3 motors. J Cell Biol 174:1035-1045.

Papermaster DS, Schneider BG, Besharse JC (1985) Vesicular transport of newly synthesized opsin from the Golgi apparatus toward the rod outer segment. Ultrastructural immunocytochemical and autoradiographic evidence in Xenopus retinas. Invest Ophthalmol Vis Sci 26:1386-1404.

Pazour GJ, Wilkerson CG, Witman GB (1998) A dynein light chain is essential for the retrograde particle movement of intraflagellar transport (IFT). J Cell Biol 141:979-992.

Pazour GJ, Dickert BL, Vucica Y, Seeley ES, Rosenbaum JL, Witman GB, Cole DG (2000) Chlamydomonas IFT88 and its mouse homologue, polycystic kidney disease gene tg737, are required for assembly of cilia and flagella. J Cell Biol 151:709-718.

Pazour GJ, Baker SA, Deane JA, Cole DG, Dickert BL, Rosenbaum JL, Witman GB, Besharse JC (2002) The intraflagellar transport protein, IFT88, is essential for vertebrate photoreceptor assembly and maintenance. J Cell Biol 157:103-113.

Peters KR, Palade GE, Schneider BG, Papermaster DS (1983) Fine structure of a periciliary ridge complex of frog retinal rod cells revealed by ultrahigh resolution scanning electron microscopy. J Cell Biol 96:265-276.

Piperno G, Mead K (1997) Transport of a novel complex in the cytoplasmic matrix of Chlamydomonas flagella. Proc Natl Acad Sci USA 94:4457-4462.

Qin H, Burnette DT, Bae YK, Forscher P, Barr MM, Rosenbaum JL (2005) Intraflagellar transport is required for the vectorial movement of TRPV channels in the ciliary membrane. Curr Biol 15:1695-1699.

Redmond TM, Yu S, Lee E, Bok D, Hamasaki D, Chen N, Goletz P, Ma JX, Crouch RK, Pfeifer K (1998) Rpe65 is necessary for production of 11cis-vitamin A in the retinal visual cycle. Nat Genet 20:344-351.

Röhlich P (1975) The sensory cilium of retinal rods is analogous to the transitional zone of motile cilia. Cell Tissue Res 161:421-430.

Rosenbaum JL, Witman GB (2002) Intraflagellar transport. Nat Rev Mol Cell Biol 3:813-825.

Scholey JM (2003) Intraflagellar transport. Annu Rev Cell Dev Biol 19:423-443.

Scholey JM (2008) Intraflagellar transport motors in cilia: moving along the cell's antenna. J Cell Biol 180:23-29.

Sedmak T, Wolfrum U (2010) Intraflagellar transport molecules in ciliary and nonciliary cells of the retina. J Cell Biol 189:171-186.

Snow JJ, Ou G, Gunnarson AL, Walker MR, Zhou HM, Brust-Mascher I, Scholey JM (2004) Two anterograde intraflagellar transport motors cooperate to build sensory cilia on C. elegans neurons. Nat Cell Biol 6:1109-1113.

Sprague BL, McNally JG (2005) FRAP analysis of binding: proper and fitting. Trends Cell Biol 15:84-91.

Sukumaran S, Perkins BD (2009) Early defects in photoreceptor outer segment morphogenesis in zebrafish ift57, ift 88 and ift172 intraflagellar transport mutants. Vision Res 49:479-489.

Tsujikawa M, Malicki J (2004) Intraflagellar transport genes are essential for differentiation and survival of vertebrate sensory neurons. Neuron 42:703-716.

Usukura J, Bok D (1987) Changes in the localization and content of opsin during retinal development in the rds mutant mouse: immunocytochemistry and immunoassay. Exp Eye Res 45:501-515.

Wey CL, Cone RA, Edidin MA (1981) Lateral diffusion of rhodopsin in photoreceptor cells measured by fluorescence photobleaching and recovery. Biophys J 33:225-232.

Whitehead JL, Wang SY, Bost-Usinger L, Hoang E, Frazer KA, Burnside B (1999) Photoreceptor localization of the KIF3A and KIF3B subunits of the heterotrimeric microtubule motor kinesin II in vertebrate retina. Exp Eye Res 69:491-503.

Young RW (1967) The renewal of photoreceptor cell outer segments. J Cell Biol 33:61-72.

Young RW (1968) Passage of newly formed protein through the connecting cilium of retina rods in the frog. J Ultrastruct Res 23:462-473. 